\title{
Daphnetin Protects against Cerebral Ischemia/Reperfusion Injury in Mice via Inhibition of TLR4/NF- $\kappa$ B Signaling Pathway
}

\author{
Jia Liu, Qianxue Chen, Zhihong Jian, Xiaoxing Xiong, Lingmin Shao, Tong Jin, \\ Xiqun Zhu, and Lei Wang
}

Department of Neurosurgery, Renmin Hospital of Wuhan University, Wuhan, Hubei 430060, China

Correspondence should be addressed to Qianxue Chen; chenqx666@sohu.com

Received 10 September 2016; Accepted 13 November 2016

Academic Editor: Gelin Xu

Copyright (c) 2016 Jia Liu et al. This is an open access article distributed under the Creative Commons Attribution License, which permits unrestricted use, distribution, and reproduction in any medium, provided the original work is properly cited.

\begin{abstract}
Growing evidences indicate that immune-mediated mechanisms contribute to the development of cerebral ischemia/reperfusion (I/R) injury. Daphnetin (DAP) is a coumarin derivative extracted from Daphne odora var., which displays anti-inflammatory properties. However, the effect of DAP on cerebral I/R injury is not yet clear. Recent studies have demonstrated that TLR4/NF$\kappa \mathrm{B}$ signaling pathway takes part in the damaging inflammatory process of cerebral $\mathrm{I} / \mathrm{R}$ injury. The present study aimed to investigate the effect of DAP on cerebral $\mathrm{I} / \mathrm{R}$ injury in vivo and its possible mechanisms. DAP was administered before middle cerebral artery occlusion and reperfusion in mice. The neurological scores, cerebral infarct sizes, the levels of inflammatory cytokines, apoptotic neural cells, and the levels of TLR4, NF- $\kappa \mathrm{B}$ p65, and $\mathrm{I} \kappa \mathrm{B} \alpha$ were estimated. The results showed that an obvious improvement of neurological scores and infarct sizes was observed in DAP-treated mice after MCAO/R. DAP treatment decreased the overexpression of TNF- $\alpha$, IL-1 $\beta$, and IL- 6 and attenuated neural cells apoptosis. Moreover, DAP treatment decreased the TLR4 expression, I $\kappa \mathrm{B}-\alpha$ degradation, and nuclear translocation of NF- $\kappa \mathrm{B}$. Taken together, our results suggested that DAP exerted neuroprotective and anti-inflammatory effects on cerebral I/R injury. The potential mechanism was involved in the inhibition of TLR4/NF- $\kappa$ B mediated inflammatory signaling pathway.
\end{abstract}

\section{Introduction}

Ischemic stroke is a leading cause of mortality and disability in adults worldwide [1]. Administration of intravenous thrombolysis with tissue plasminogen activator $(\mathrm{t}-$ PA) is currently approved for the treatment of ischemic stroke. However, a secondary impairment known as cerebral ischemia/reperfusion $(\mathrm{I} / \mathrm{R})$ injury comes up with the $\mathrm{t}-\mathrm{PA}$ treatment [2]. It results from a complex pattern of pathophysiological events. The critical role of immune-mediated mechanisms in cerebral I/R injury has been increasingly recognized in the last decades [3] and the important contribution of Toll-like receptors (TLRs) in the induction of inflammatory responses has been demonstrated [4]. Toll-like receptor 4 (TLR4) was found to be highly induced after cerebral I/R [5]. In addition, it has been reported that TLR4/NF- $\kappa \mathrm{B}$ signaling pathway takes part in the damaging inflammatory process of cerebral I/R injury [6], and inhibiting TLR4/NF- $\kappa$ B signaling pathway can downregulate inflammation and alleviate brain injury [7]. Therefore, TLR4/NF- $\kappa$ B signaling pathway may be a therapeutic target for cerebral I/R injury.

At present, lots of studies focus on exploring antiinflammatory and neuroprotective agents that may alleviate cerebral I/R injury. Natural products probably represent an ideal source to develop safe and effective agents for management of cerebral I/R injury. Daphnetin (DAP, Figure 1), a coumarin derivative extracted from Daphne odora var., is a natural anti-inflammatory product $[8,9]$. The traditional Chinese medicine Zushima, primarily composed of daphnetin, has been used in the treatment of inflammatory diseases. Recent studies showed that DAP could provide anti-inflammatory effects in rheumatoid arthritis [10, 11], endotoxin-induced lung injury [12], and acute pancreatitis [13]. DAP inhibits the infiltration of inflammatory cells and alleviates the inflammatory injury state in mice through decreasing the production of proinflammatory cytokines, including tumor necrosis factor- $\alpha$ (TNF- $\alpha$ ), interleukin- $1 \beta$ (IL-1 $\beta$ ), and interleukin-6 (IL-6) [10-12]. More importantly, 


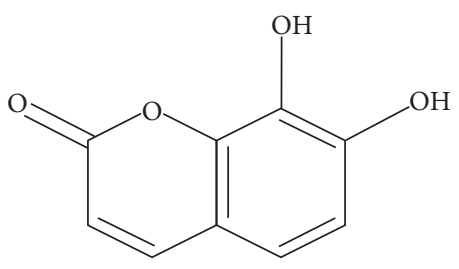

Figure 1: The chemical structure of daphnetin.

novel findings suggested that DAP had neuroprotective effects in experimental autoimmune encephalomyelitis [14] and prevented chronic unpredictable stress-induced cognitive deficits [15]. However, the neuroprotective effect of DAP on cerebral I/R injury is not yet clear and the ability of DAP to antagonize the inflammatory signaling pathway in brain remains to be determined.

Therefore, given the functional relevance of DAP in inflammatory diseases and neuropathology, we supposed that DAP protected against cerebral I/R injury via downregulation of TLR4/NF- $\kappa \mathrm{B}$ signaling pathway. We sought to explore the potential role of DAP in cerebral I/R injury and the involving mechanisms.

\section{Materials and Methods}

2.1. Animals. C57BL/6 mice (23-28g) were obtained from the Center of Experimental Animals, Wuhan University, Hubei, China. The mice were raised in a $12 \mathrm{~h}$ dark-light cycle circumstance with food and water available. All experimental procedures involving animals were in accordance with the Guide for the Care and Use of Laboratory Animals by National Institutes of Health and the protocol was approved by the Animal Experimentation Ethics Committee of Wuhan University.

\subsection{Preparation of Middle Cerebral Artery Occlusion and} Reperfusion (MCAO/R) Model. The mouse was anesthetized with $5.0 \%$ isoflurane and maintained on $1.5 \%$ isoflurane in $70 \% \mathrm{~N}_{2} \mathrm{O}$ and $30 \% \mathrm{O}_{2}$ using a small animal anesthesia system. It was placed on an operating table in a supine position. The surgery was carried out under an operating microscope. The left carotid triangle, common carotid artery (CCA), carotid bifurcation, external carotid artery (ECA) and internal carotid artery (ICA) were exposed with blunt dissection. The CCA was temporarily ligated and the ECA was permanently ligated with a $6 / 0$ suture. Then, the ECA was coagulated and cut distal to the ligature and retracted caudally. Two temporary ligatures were prepared around the ICA: one distal to the bifurcation and the other exactly above the bifurcation. An arteriotomy was performed in the ECA stump and a monofilament suture was inserted. The monofilament suture was slowly advanced to reach the terminal ICA until a mild resistance was felt and the ligature on the ICA was tightened. Then, the incision was temporarily closed and the mouse was placed back in the cage. One hour after the occlusion, the mouse was reanesthetized and the monofilament was gently removed from the MCA. The hole was closed with electrocautery and normal blood flow perfusion was reestablished. After recovery from anesthesia, the mouse was returned to the cage and raised at $23 \pm 1^{\circ} \mathrm{C}$.

2.3. Experimental Groups and Daphnetin Treatment. Mice were randomly assigned into four groups as follows $(n=12)$ : sham operation group, $\mathrm{MCAO} / \mathrm{R}$ group, $\mathrm{MCAO} / \mathrm{R}+$ vehicle group, and $\mathrm{MCAO} / \mathrm{R}+\mathrm{DAP}$ group. Daphnetin with purity $>$ 99.4\% was obtained from Tauto Biotech Co., Ltd. (Shanghai, China) and diluted in $0.9 \%$ saline. Daphnetin solution was administered via intraperitoneal injection 30 minutes before $\mathrm{MCAO} / \mathrm{R}$. In the preliminary experiment, mice received three different doses of DAP $(5,10$, and $20 \mathrm{mg} / \mathrm{kg})$. An equal volume of $0.9 \%$ saline was administered in the $\mathrm{MCAO} / \mathrm{R}+$ vehicle group.

2.4. Evaluation of Neurological Deficit. Neurological deficit was evaluated by an investigator blinded to the experimental groups after $24 \mathrm{~h}$ reperfusion. The scoring system was based on a 5-point scale system described previously [16]: no neurological deficit, 0 ; failure to extend right forepaw fully, 1 ; circling to the right, 2; falling to the right, 3; inability to walk spontaneously and depressed levels of consciousness, 4 .

2.5. Assessment of Cerebral Infarction. The effect of DAP on cerebral infarct volume was assessed by 2,3,5-Triphenyltetrazolium Chloride (TTC) Staining. Following cerebral ischemia/reperfusion injury for 24 hours, mice were euthanized under deep anesthesia with 10\% chloral hydrate. The brains were quickly removed and frozen at $-20^{\circ} \mathrm{C}$ for $10 \mathrm{~min}$. Then, coronal sections with a $2 \mathrm{~mm}$ thickness were cut and the slices were stained with $2 \%$ TTC solution (Sigma, St. Louis, MO, USA) for 20 minutes at $37^{\circ} \mathrm{C}$ followed by fixation with $4 \%$ paraformaldehyde overnight. The normal brain tissue was stained dark red, whereas the infarcted area remained pale white. The infarct area was analyzed by an observer, blinded to the experimental conditions, using ImageJ analysis software $(1.37 \mathrm{v} \mathrm{NIH})$ and the percentage of infarction volume was calculated as described previously [17].

2.6. ELISA to Determine Inflammatory Cytokines. The levels of inflammatory cytokines (TNF- $\alpha$, IL-1 $\beta$, and IL-6) were quantified using ELISA assay kits (Boster, Hubei, China). The experiments were conducted according to the manufacturer's instructions.

2.7. TUNEL Assay to Determine Neural Cells Apoptosis. The formalin-fixed tissue was embedded in paraffin and sectioned at $4 \mu \mathrm{m}$ thickness with a microtome. The sections were analyzed by the TUNEL assay to detect the apoptotic cells. The TUNEL assay kit was purchased from Roche Molecular Biochemicals, Mannheim, Germany, and the experiment was conducted according to manufacturer's protocol. Apoptotic cells with condensed nuclei were stained brown in the TUNEL assay, while normal cells were large, round, and not stained. The positive cells were analyzed under a light microscope by an investigator blinded to the experimental groups. The extent of brain injury was evaluated by the percentage of TUNEL-positive cells. 


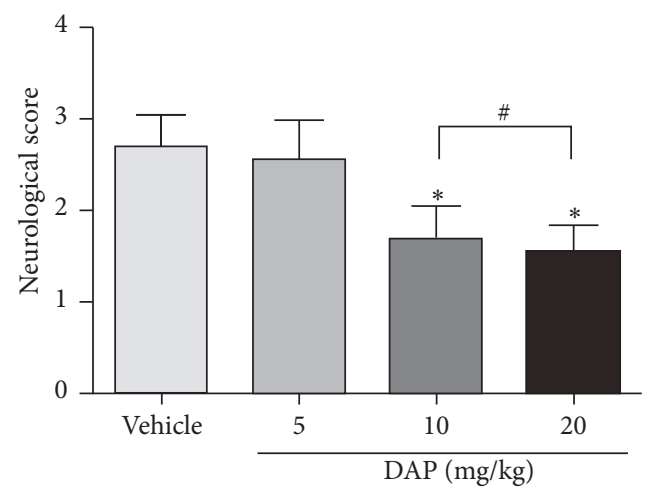

(a)
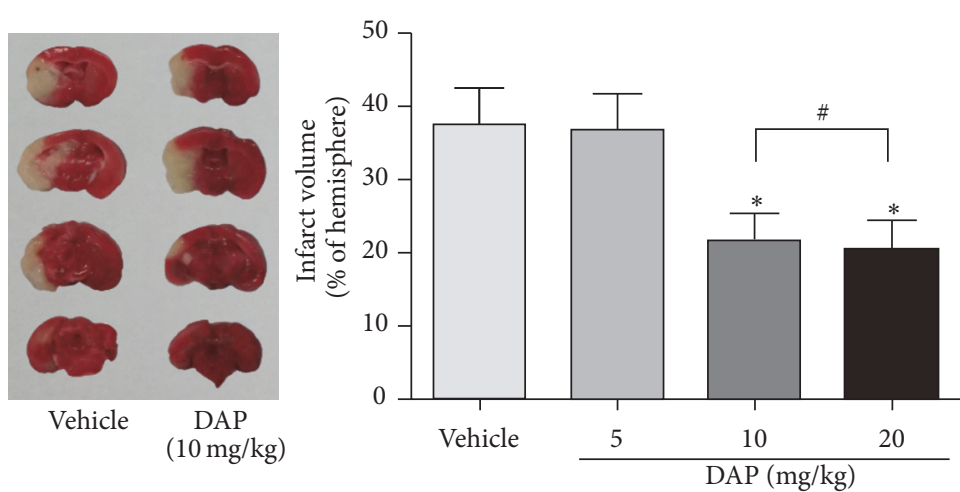

(b)

FIGURE 2: Effect of DAP on neurological deficit and infarct size after MCAO/R. ${ }^{*} P<0.05$ versus the vehicle group; ${ }^{\#} P>0.05$ for no statistical significance between $10 \mathrm{mg} / \mathrm{kg}$ and $20 \mathrm{mg} / \mathrm{kg}$ DAP treatment group.

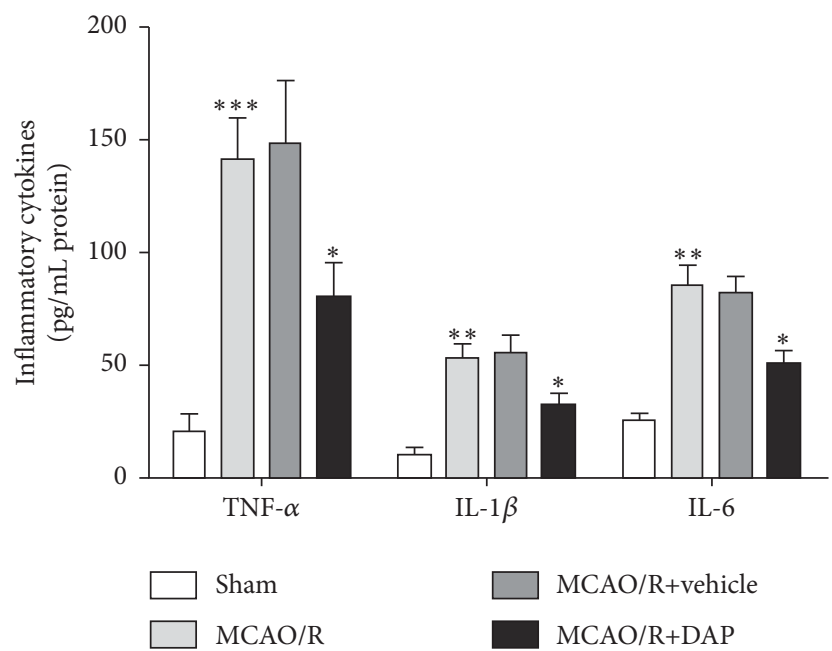

FIGURE 3: Effect of DAP on the levels of proinflammatory cytokines in the brain tissue after MCAO/R. ${ }^{*} P<0.05$ versus the vehicle group; ${ }^{* *} P<0.01$ versus the sham group; ${ }^{* * *} P<0.001$ versus the sham group.

2.8. Western Blot Analysis. Total proteins were extracted from the infarct cortex and protein concentrations were determined using a BCA assay kit as instructed by the manufacturer (Beyotime Biotech, Jiangsu, China). 1/4 volume of $5 \mathrm{x}$ loading buffer was added and followed by a boiling water bath for 10 minutes for denaturation. Samples, containing $40 \mu \mathrm{g}$ proteins, were loaded and separated by electrophoresis on $12 \%$ SDS-polyacrylamide gels. Subsequently, proteins were transferred onto PVDF membranes, blocked for two hours with $5 \%$ nonfat dry milk at room temperature, and incubated with rabbit anti-TLR4 (1:300, Abcam, Cambridge, MA, USA), anti-NF- $\kappa$ B p65 (1: 2000, Proteintech, Rosemont, USA), anti-I $\kappa \mathrm{B} \alpha$ (1:2000, Proteintech, Rosemont, USA), anti- $\beta$-actin ( $1: 200$, Boster, Hubei, China), and anti-Histone 3 (1:2000, Proteintech, Rosemont, USA) antibodies at $4^{\circ} \mathrm{C}$ on a shaker overnight and then washed, followed by incubation with the corresponding HRP-conjugated secondary antibody (Boster, Hubei, China). Finally, ECL solution was added to reveal the bands and the gray value was analyzed by ImageJ software (NIH).

2.9. Statistical Analysis. Data was presented as mean \pm SD. Differences between the mean values were evaluated by Student's $t$-test or one-way ANOVA test. For all tests, values of $P<0.05$ were considered to be statistically significant. Statistical analyses were performed using SPSS 17.0 software.

\section{Results}

3.1. Effects of DAP on the Neurological Score and Infarct Size after MCAO/R. MCAO/R models were established. To determine the neuroprotective effects of DAP against cerebral $\mathrm{I} / \mathrm{R}$ injury, we assessed the neurological scores and cerebral infarct sizes 24 hours after MCAO/R (Figure 2). $5 \mathrm{mg} / \mathrm{kg}$ of DAP did not affect the neurologic scores or infarct sizes compared with the vehicle $(P>0.05)$. However, the neurologic scores were significantly improved via $10 \mathrm{mg} / \mathrm{kg}$ and $20 \mathrm{mg} / \mathrm{kg}$ of DAP treatment $(P<0.05$, Figure $2(\mathrm{a}))$. Meanwhile, as demonstrated by the TTC-stained coronal sections, the infarct sizes were also reduced at the same doses compared with the vehicle $(P<0.05$, Figure $2(\mathrm{~b}))$. In addition, $10 \mathrm{mg} / \mathrm{kg}$ and $20 \mathrm{mg} / \mathrm{kg}$ of DAP protected against cerebral I/R injury to the same degree in this model $(P>$ 0.05). Based on these results and the previous study $[14,15]$, the optimal treatment dose of DAP $(10 \mathrm{mg} / \mathrm{kg})$ was used in the following experiment.

3.2. Effects of DAP on the Expression of TNF- $\alpha, I L-1 \beta$, and IL-6 after $M C A O / R$. To identify the downstream effectors in the TLR4/NF- $\kappa$ B signaling pathway, the levels of inflammatory cytokines (TNF- $\alpha$, IL-1 $\beta$, and IL-6) were examined by ELISA (Figure 3 ). The results demonstrated that TNF- $\alpha$, IL-1 $\beta$, and IL-6 were robustly expressed in the sham group $(P<0.001 ; P<0.01)$. However, DAP administration significantly decreased the levels of TNF- $\alpha$, IL- $1 \beta$, and IL- 6 

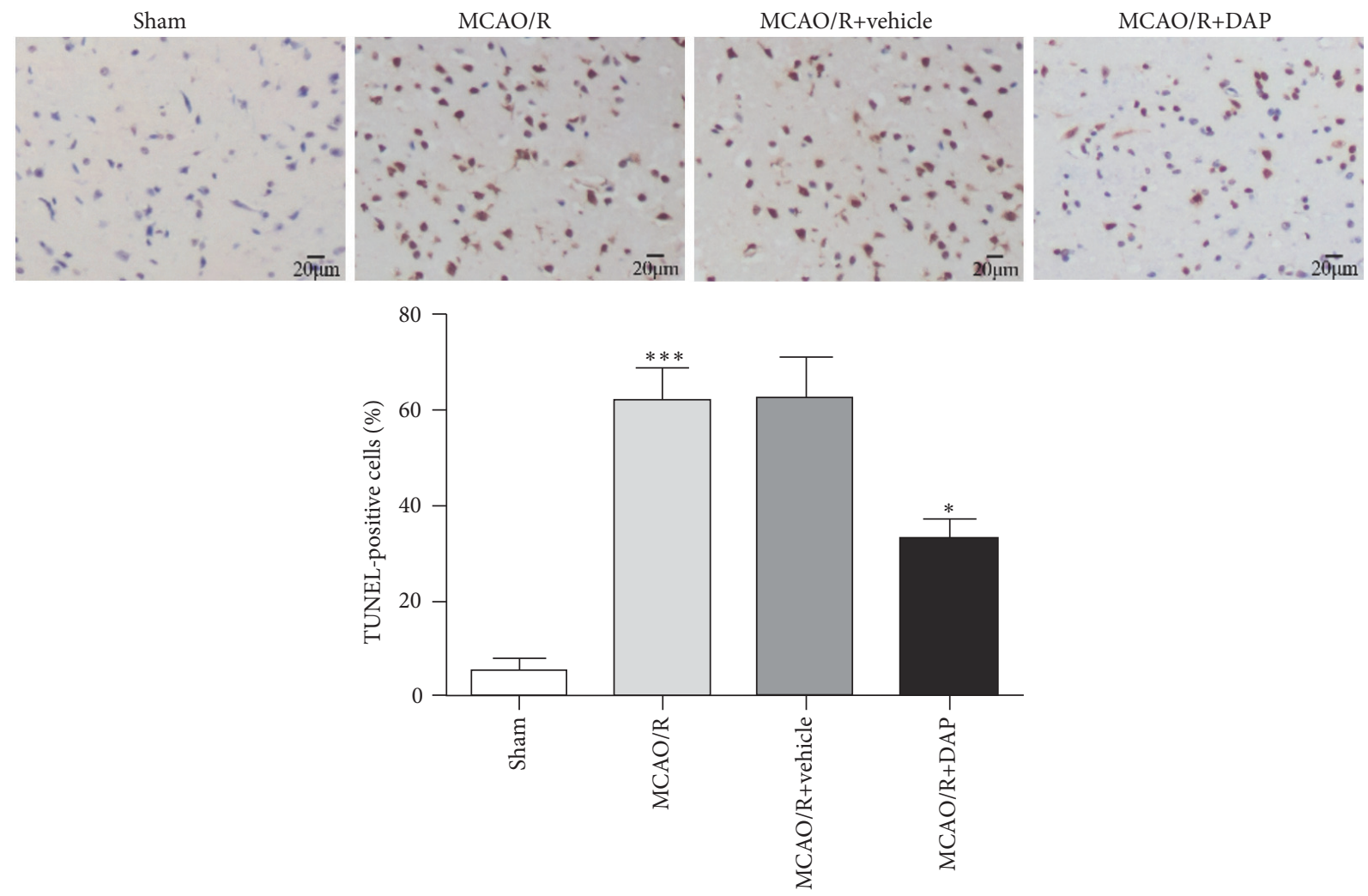

Figure 4: Effect of DAP on neural cells apoptosis, based on the TUNEL assay. Scale bars: $20 \mu \mathrm{m} .{ }^{*} P<0.05$ versus the vehicle group; ${ }^{* * *} P<$ 0.05 versus the sham group.

$(P<0.05)$. These findings suggested that DAP suppressed brain inflammation responses after stroke.

3.3. Effects of DAP on Neural Cells Apoptosis after MCAO/R. To further evaluate the effect of DAP on neural cells apoptosis after cerebral I/R injury, we examined apoptotic cells death using the TUNEL assay. As shown in Figure 4, we found that the number of apoptotic cells in the MCAO/R group was significantly increased compared with the sham group $(P<0.001)$. However, the number of apoptotic cells was significantly decreased in the DAP treatment group compared with the vehicle group $(P<0.05)$, suggesting the antiapoptotic activity of DAP in cerebral I/R injury.

3.4. Effects of Daphnetin on the TLR4/NF- $\kappa B$ Signaling Pathway in MCAO/R Mice. To provide evidence regarding the contribution of TLR $4 / \mathrm{NF}-\kappa \mathrm{B}$ signaling pathway to the protective effects of DAP treatment, the TLR4, NF- $\kappa \mathrm{B}$ p 65 , and $\mathrm{I} \kappa \mathrm{B} \alpha$ protein levels were measured by Western blot analysis (Figure 5). The protein levels of TLR 4 and NF- $\kappa$ B p 65 in the $\mathrm{MCAO} / \mathrm{R}$ group were abundantly increased compared with the sham group $(P<0.05 ; P<0.01)$, whereas TLR4 and NF- $\kappa \mathrm{B}$ p65 in the DAP treatment group were dramatically decreased compared with the vehicle group $(P<$ 0.05). Furthermore, DAP treatment remarkably decreased the degradation of $\mathrm{I} \kappa \mathrm{B} \alpha(P<0.05)$. These results suggested that the TLR $4 / \mathrm{NF}-\kappa \mathrm{B}$ signaling pathway was involved with the protective effects of DAP on cerebral I/R injury.

\section{Discussion}

The results of the present study demonstrated that DAP showed neuroprotective effects on cerebral I/R injury in $\mathrm{MCAO} / \mathrm{R}$ mice. An obvious improvement of neurological deficit scores and infarct sizes was observed in DAP-treated mice 24 hours after reperfusion. Meanwhile, DAP decreased the overexpression of TNF- $\alpha$, IL- $1 \beta$, and IL- 6 and attenuated neural cells apoptosis. In addition, we evaluated the effects of DAP on the expression of TLR 4 , NF- $\kappa \mathrm{B}$ p 65 , and $\mathrm{I} \kappa \mathrm{B} \alpha$, which indicated that the underlying mechanism of the neuroprotective effects was involved with the inhibition of the TLR4/NF- $\kappa \mathrm{B}$ signaling pathway.

Cerebral ischemia/reperfusion injury is a severe clinical problem with high mortality and morbidity. More recently, accumulating evidences suggest that immune-mediated mechanisms play a critical role in the secondary brain damage following cerebral I/R $[3,18]$. Immune-mediated mechanisms emerge as a new frontier and need more consideration in devising therapeutic strategy for improving cerebral I/R injury outcomes. Inhibiting inflammatory responses has been a therapeutic target for cerebral I/R injury.

Daphnetin, a natural coumarin derivative isolated from the traditional Chinese medicinal herb Daphne odora var., has been reported to show anti-inflammatory properties $[8,9,19]$. Recent studies show that DAP has protective effects in inflammatory diseases and neuropathology [1015]. However, the neuroprotective effect of DAP on cerebral 


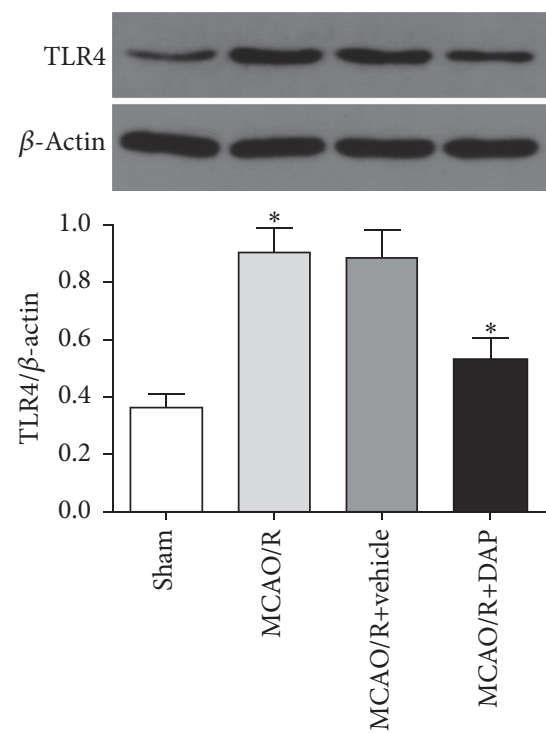

(a)
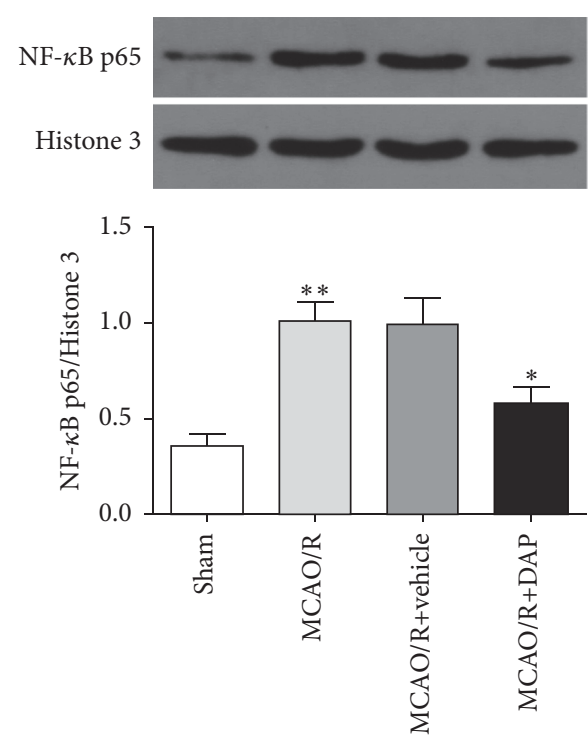

(b)
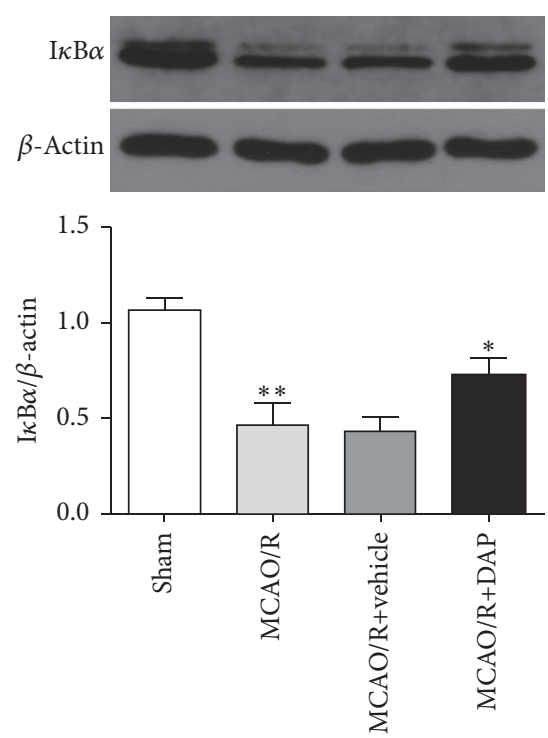

(c)

Figure 5: Effect of DAP on the protein levels of TLR4, NF- $\kappa \mathrm{B}$ p65, and $\mathrm{I} \kappa \mathrm{B} \alpha$, based on Western blot analysis. (a) ${ }^{*} P<0.05$ versus the sham group or the vehicle group. ((b) and (c)) ${ }^{*} P<0.05$ versus the vehicle group; ${ }^{* *} P<0.01$ versus the sham group.

$\mathrm{I} / \mathrm{R}$ injury has not been clear and the ability of DAP to antagonize the inflammatory signaling pathway remains to be determined. In order to develop cerebral I/R injury in mice, we established the cerebral I/R mice model induced by $\mathrm{MCAO} / \mathrm{R}$ [20]. The evaluation of the neurological score and cerebral infarct size indicated that DAP treatment effectively protected against cerebral $\mathrm{I} / \mathrm{R}$ injury induced by $\mathrm{MCAO} / \mathrm{R}$. $10 \mathrm{mg} / \mathrm{kg}$ and $20 \mathrm{mg} / \mathrm{kg}$ of DAP protected against cerebral I/R injury to the same degree, whereas $5 \mathrm{mg} / \mathrm{kg}$ of DAP did not show neuroprotective effects. According to this result and the previous study [14, 15], $10 \mathrm{mg} / \mathrm{kg}$ was chosen as the optimal treatment dose in the subsequent experiment.

In the inflammation responses following cerebral $I / R$ injury, glial cells are activated and peripheral immune cells infiltrate into brain, resulting in the secretion of a great number of inflammatory cytokines which induce neural cells apoptosis. TNF- $\alpha$, IL-1 $\beta$, and IL- 6 are the potential inflammatory cytokines which are involved in that progression and brain with cerebral I/R injury was observed with increased levels of TNF- $\alpha$, IL- $1 \beta$, and IL-6 $[21,22]$. In the present study, our results showed that DAP remarkably decreased the levels of TNF- $\alpha$, IL-1 $\beta$, and IL- 6 and attenuated the apoptosis of neural cells. Therefore, DAP suppressed brain inflammation responses after stroke. We explored the potential mechanism underlying its neuroprotective and anti-inflammatory effects as well in the following studies.

The activation of innate immune receptors such as Tolllike receptors (TLRs) has been demonstrated to play an important role in the induction of inflammatory responses $[3,18]$. TLRs represent a family of transmembrane patternrecognition receptors, which can be activated by endogenous damage-associated molecular patterns (DAMPs) released from injured or stressed cells under ischemic situation. TLR4 was the first mammalian TLR recognized. DAMPs can activate the TLR4/NF-kB signaling pathway in the neighboring microglia and induce the release of proinflammatory cytokines, which leads to the degeneration and apoptosis of neural cells [23]. Recent studies have also shown that TLR4 is highly induced after cerebral $I / R$ and cerebral $\mathrm{I} / \mathrm{R}$ injury is alleviated in TLR4-deficient mice $[5,24]$. All these results demonstrate that TLR4/NF- $\kappa \mathrm{B}$ signaling pathway is involved in cerebral I/R injury. Therefore, we supposed that DAP may protect against cerebral I/R injury by inhibiting TLR4/NF- $\kappa \mathrm{B}$ mediated inflammatory signaling pathway. In the present study, we found that cerebral I/R injury was associated with a significant increase of TLR4 expression, I $\kappa \mathrm{B}-\alpha$ degradation, and nuclear translocation of NF- $\kappa B$, which were consistent with the previous studies. However, DAP treatment dramatically decreased the TLR4 expression, I $\kappa \mathrm{B}-\alpha$ degradation, and nuclear translocation of NF- $\kappa$ B. These results suggested that DAP inhibited TLR4/NF- $\kappa \mathrm{B}$ mediated inflammatory signaling pathway in cerebral I/R injury. Taken together, our results indicated that the TLR4/NF- $\kappa \mathrm{B}$ signaling pathway was involved with the neuroprotective and anti-inflammatory effects of DAP on cerebral I/R injury in mice.

\section{Conclusion}

In summary, the present study demonstrated that DAP alleviated cerebral $\mathrm{I} / \mathrm{R}$ injury by reducing inflammatory cytokines production and neural cells apoptosis in vivo. Furthermore, the potential mechanism responsible for neuroprotective and anti-inflammatory effects of DAP was involved with the inhibition of TLR4/NF- $\kappa$ B mediated inflammatory signaling pathway. DAP showed a therapeutic potential for the treatment of stroke. 


\section{Competing Interests}

The authors declare that there are no competing interests regarding the publication of this paper.

\section{Acknowledgments}

This work was supported by grants from National Natural Science Foundation of China (81372683).

\section{References}

[1] V. L. Feigin, "Stroke epidemiology in the developing world," Lancet, vol. 365, no. 9478, pp. 2160-2161, 2005.

[2] C. H. Y. Wong and P. J. Crack, "Modulation of neuroinflammation and vascular response by oxidative stress following cerebral ischemia-reperfusion injury," Current Medicinal Chemistry, vol. 15, no. 1, pp. 1-14, 2008.

[3] C. Iadecola and J. Anrather, "The immunology of stroke: from mechanisms to translation," Nature Medicine, vol. 17, no. 7, pp. 796-808, 2011.

[4] H. K. Eltzschig and T. Eckle, "Ischemia and reperfusion-from mechanism to translation," Nature Medicine, vol. 17, no. 11, pp. 1391-1401, 2011

[5] J. R. Caso, J. M. Pradillo, O. Hurtado, P. Lorenzo, M. A. Moro, and I. Lizasoain, "Toll-like receptor 4 is involved in brain damage and inflammation after experimental stroke," Circulation, vol. 115, no. 12, pp. 1599-1608, 2007.

[6] F. Hua, J. Ma, T. Ha et al., "Activation of Toll-like receptor 4 signaling contributes to hippocampal neuronal death following global cerebral ischemia/reperfusion," Journal of Neuroimmunology, vol. 190, no. 1-2, pp. 101-111, 2007.

[7] P.-F. Wang, H. Fang, J. Chen et al., "Polyinosinic-polycytidylic acid has therapeutic effects against cerebral ischemia/ reperfusion injury through the downregulation of TLR4 signaling via TLR3," Journal of Immunology, vol. 192, no. 10, pp. 47834794, 2014.

[8] K. C. Fylaktakidou, D. J. Hadjipavlou-Litina, K. E. Litinas, and D. N. Nicolaides, "Natural and synthetic coumarin derivatives with anti-inflammatory/antioxidant activities," Current Pharmaceutical Design, vol. 10, no. 30, pp. 3813-3833, 2004.

[9] K. N. Venugopala, V. Rashmi, and B. Odhav, "Review on natural coumarin lead compounds for their pharmacological activity," BioMed Research International, vol. 2013, Article ID 963248, 14 pages, 2013.

[10] R. Yao, Y. Fu, S. Li, L. Tu, X. Zeng, and N. Kuang, "Regulatory effect of daphnetin, a coumarin extracted from Daphne odora, on the balance of Treg and Th17 in collagen-induced arthritis," European Journal of Pharmacology, vol. 670, no. 1, pp. 286-294, 2011.

[11] L. Tu, S. Li, Y. Fu et al., "The therapeutic effects of daphnetin in collagen-induced arthritis involve its regulation of Th17 cells," International Immunopharmacology, vol. 13, no. 4, pp. 417-423, 2012.

[12] W.-W. Yu, Z. Lu, H. Zhang et al., "Anti-inflammatory and protective properties of daphnetin in endotoxin-induced lung injury," Journal of Agricultural and Food Chemistry, vol. 62, no. 51, pp. 12315-12325, 2014.

[13] Z.-Y. Liu, J. Liu, K.-L. Zhao et al., "Protective effects of daphnetin on sodium taurocholate-induced severe acute pancreatitis in rats," Molecular Medicine Reports, vol. 9, no. 5, pp. 1709-1714, 2014.
[14] D. Wang, Z. Lu, H. Zhang et al., "Daphnetin alleviates experimental autoimmune encephalomyelitis via regulating dendritic cell activity," CNS Neuroscience \& Therapeutics, vol. 22, no. 7, pp. 558-567, 2016.

[15] M.-J. Liao, L.-F. Lin, X. Zhou et al., "Daphnetin prevents chronic unpredictable stress-induced cognitive deficits," Fundamental and Clinical Pharmacology, vol. 27, no. 5, pp. 510-516, 2013.

[16] X. Xiong, L. Xu, L. Wei, R. E. White, Y. Ouyang, and R. G. Giffard, "IL-4 is required for sex differences in vulnerability to focal ischemia in mice," Stroke, vol. 46, no. 8, pp. 2271-2276, 2015.

[17] X. Xiong, G. E. Barreto, L. Xu, Y. B. Ouyang, X. Xie, and R. G. Giffard, "Increased brain injury and worsened neurological outcome in interleukin- 4 knockout mice after transient focal cerebral ischemia," Stroke, vol. 42, no. 7, pp. 2026-2032, 2011.

[18] Á. Chamorro, A. Meisel, A. M. Planas, X. Urra, D. Van De Beek, and R. Veltkamp, "The immunology of acute stroke," Nature Reviews Neurology, vol. 8, no. 7, pp. 401-410, 2012.

[19] B. Song, Z. Wang, Y. Liu et al., "Immunosuppressive activity of daphnetin, one of coumarin derivatives, is mediated through suppression of NF- $\kappa \mathrm{B}$ and NFAT signaling pathways in mouse T cells," PLoS ONE, vol. 9, no. 5, Article ID e96502, 2014.

[20] Q. Cai, Z. Chen, D. K. Kong et al., "Novel microcatheterbased intracarotid delivery approach for MCAO/R mice," Neuroscience Letters, vol. 597, pp. 127-131, 2015.

[21] Y. Yasuda, T. Shimoda, K. Uno et al., “Temporal and sequential changes of glial cells and cytokine expression during neuronal degeneration after transient global ischemia in rats," Journal of Neuroinflammation, vol. 8, article no. 70, 2011.

[22] S. E. Lakhan, A. Kirchgessner, and M. Hofer, "Inflammatory mechanisms in ischemic stroke: therapeutic approaches," Journal of Translational Medicine, vol. 7, article 97, 2009.

[23] O. A. Harari and J. K. Liao, "NF- $\kappa \mathrm{B}$ and innate immunity in ischemic stroke," Annals of the New York Academy of Sciences, vol. 1207, pp. 32-40, 2010.

[24] C.-X. Cao, Q.-W. Yang, F.-L. Lv, J. Cui, H.-B. Fu, and J.-Z. Wang, "Reduced cerebral ischemia-reperfusion injury in Tolllike receptor 4 deficient mice," Biochemical and Biophysical Research Communications, vol. 353, no. 2, pp. 509-514, 2007. 

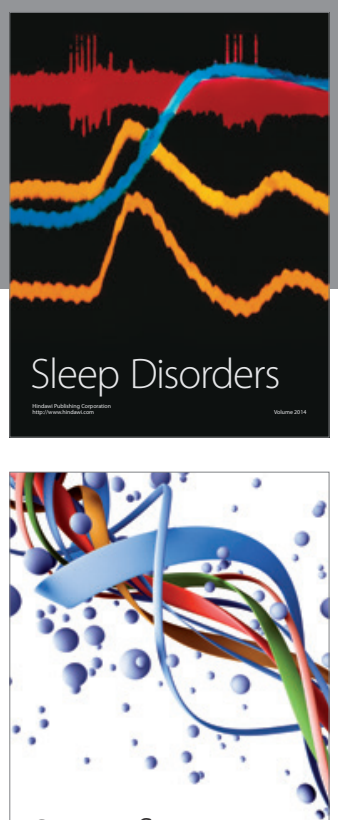

Scientifica
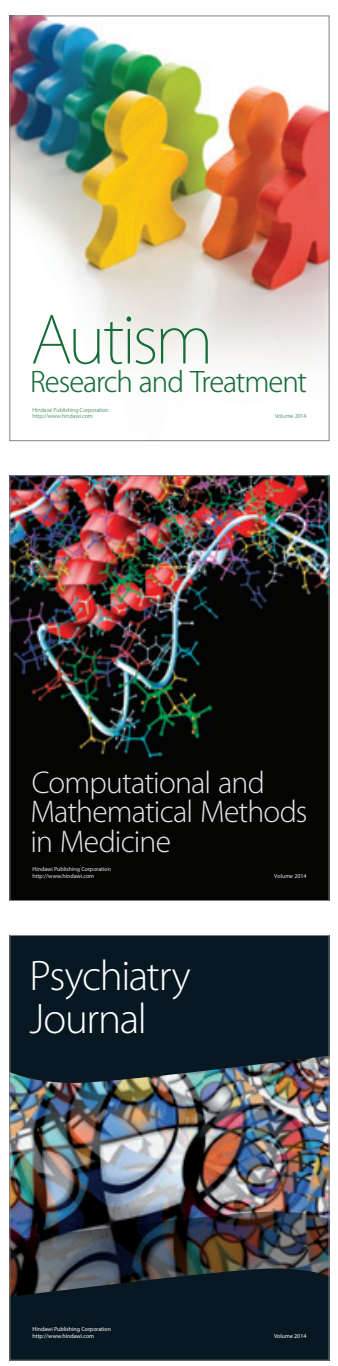
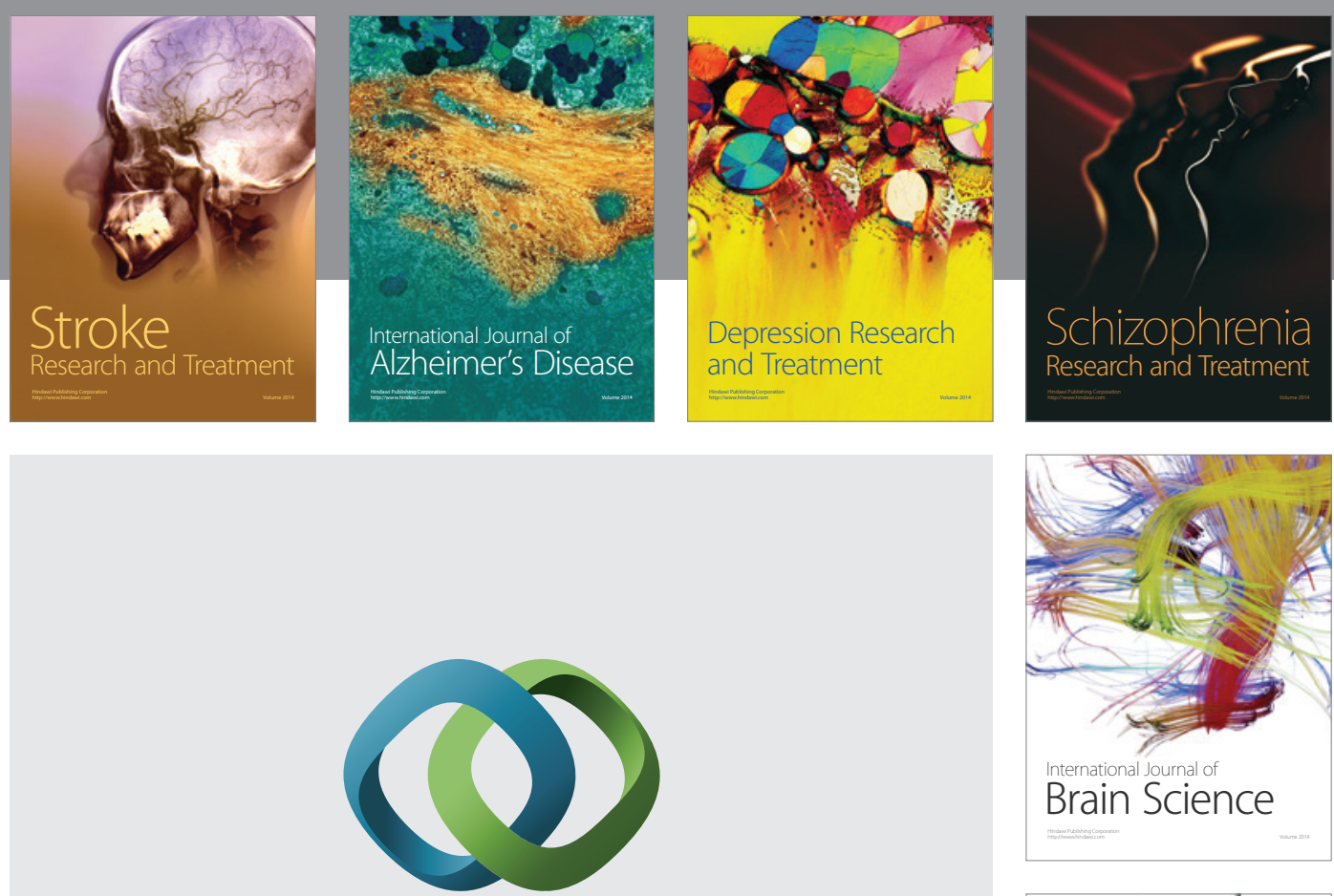

\section{Hindawi}

Submit your manuscripts at

http://www.hindawi.com
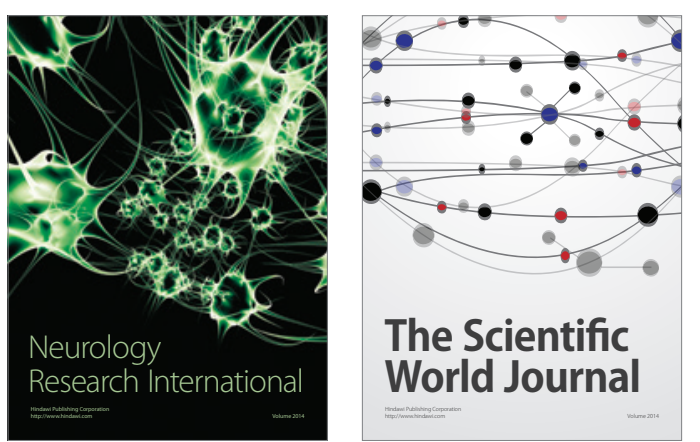

The Scientific World Journal

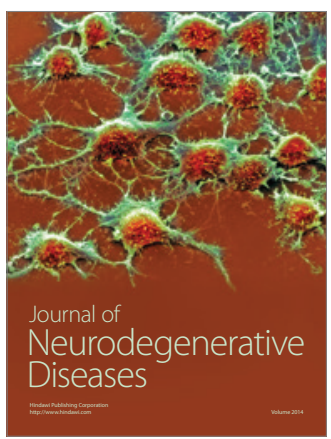

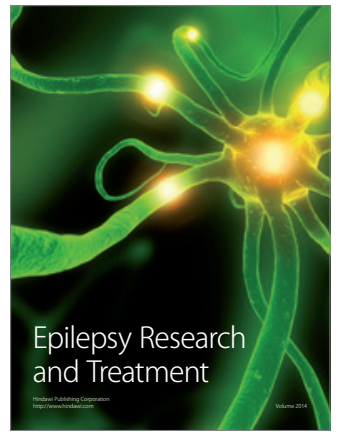

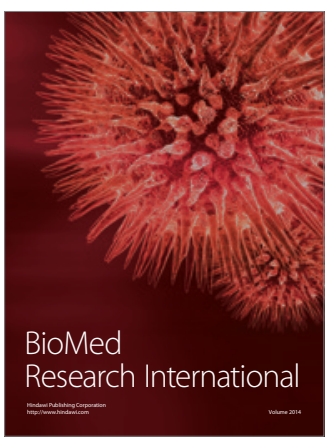

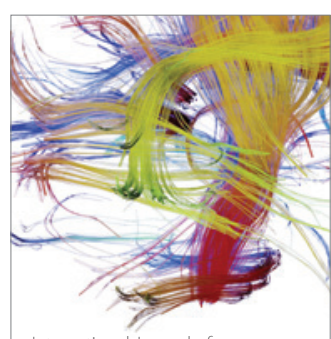

Brain Science

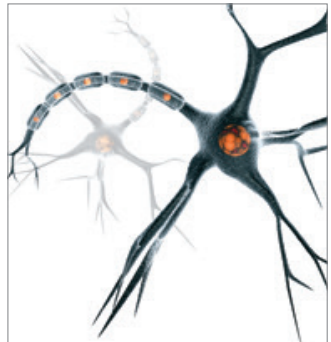

Neural Plasticity
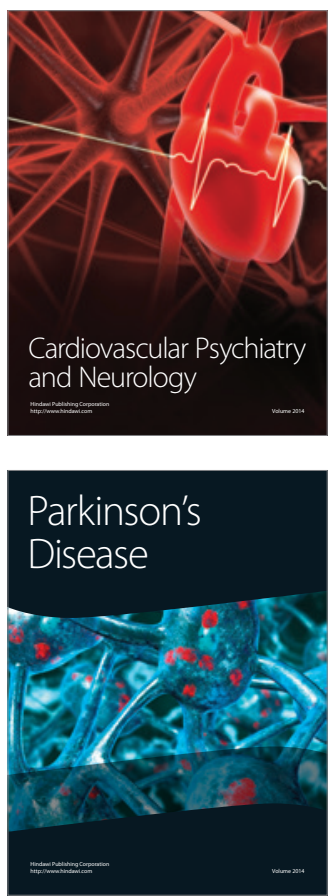